\title{
Wireless Mesh Networks: Energy - Capacity Tradeoff and Physical Layer Parameters
}

\author{
Anis Ouni \\ Université de Lyon, INRIA \\ INSA Lyon, CITI \\ F-69621, France \\ Email: anis.ouni@insa-lyon.fr
}

\author{
Hervé Rivano \\ CNRS, Université de Lyon, INRIA \\ INSA Lyon, CITI \\ F-69621, France \\ Email: Herve.Rivano@inria.fr
}

\author{
Fabrice Valois \\ Université de Lyon, INRIA \\ INSA Lyon, CITI \\ F-69621, France \\ Email: fabrice.valois@insa-lyon.fr
}

\begin{abstract}
This paper is focused on broadband wireless mesh networks based on OFDMA resource management, considering a realistic SINR model of the physical layer with a fine tuned power control at each node. A linear programing model using column generation leads to compute power efficient schedules with high network capacity. Correlation between capacity and energy consumption is analyzed as well as the impact of physical layer parameters - SINR threshold and path-loss exponent. We highlight that there is no significant tradeoff between capacity and energy when the power consumption of idle nodes is important. We also show that both energy consumption and network capacity are very sensitive to the SINR threshold variation.
\end{abstract}

Keywords-Mesh networks, capacity, energy, scheduling

\section{INTRODUCTION}

High data rate is a challenge for the next generation cellular networks. This objective needs a significative densification of cells which requires an efficient backhauling infrastructure. We consider a broadband wireless mesh network (WMN) composed of a twofold architecture: $i$ ) clients are connected to base station (BS) and ii) a wireless backhaul topology interconnects the BS with the core network. These BSs are equipped with routing functionalities and communicate together through radio links, as in LTE-Advanced relay or WIMAX Mesh. The BS collect the traffic generated by the mobile clients and forward it through multi-hop communications to some dedicated BS, denoted gateways, that bridge the backhauling network to the core network (Fig. 1). We assume that mobileto-BS and BS-to-BS traffics use different and independent resources. In this work, we focus on the backhauling network and we do not take into account the users requests but rather their flows aggregated by the BS.

Optimizing the network capacity is one of the main research issues for WMNs since the seminal work of Gupta and Kumar [1] where asymptotic capacity is linked to the size of the network. Besides, minimizing the energy expenditure and electromagnetic pollution of such infrastructures are hot societal and economical challenges nowadays (see EARTH, CARMEN european projetcs) ${ }^{1}$. The main contribution of this paper is to address the optimization of both network capacity and energy consumption.

\footnotetext{
${ }^{1}$ EARTH: https://www.ict-earth.eu/, CARMEN: http://www.ict-carmen.eu/
}

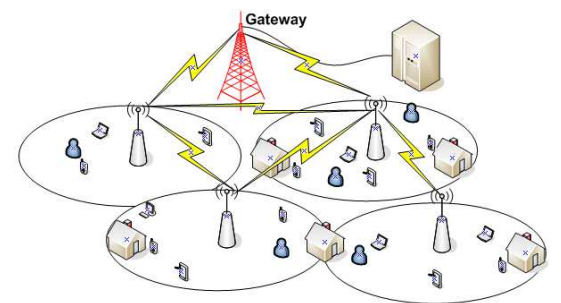

Fig. 1. Wireless mesh network architecture: base stations collect the traffic from clients (mobile or static) and forward it to the core network.

In [2], a multi-objective framework has been developed to study the tradeoff between capacity and energy consumption with a simplistic binary interference model without power control. In our work, we introduce a Signal-to-Noise-andInterference-Ratio (SINR) based model with fine tuned power control at each node. This allows us to investigate deeply on the capacity/energy tradeoff and to study the impact of physical layer parameters such as the SINR threshold and the path-loss exponent on these metrics. In our model, a column generation algorithm isolates the routing and scheduling models from the computation of concurrent links activations, and the computation of time/frequency resource allocation accounting for the energy expenditure.

The rest of paper is organized as follows. Section II reviews related works. The problem statement and the network model are discussed in Section III. Then, we present our multiobjective framework based on a linear program and a column generation. Section VI investigates the impact of physical layer parameters, and the tradeoff between energy consumption and capacity. Finally, we conclude and introduce future directions.

\section{RELATED WORK}

To increase the throughput provided to nodes, several studies have addressed the link scheduling problem, i.e. to identify sets of links that can be simultaneously activated. [3] is focused on the scheduling problem around an access point on 802.11 networks. It investigates the Round Weighting Problem (RWP) trying to determine the minimum number of rounds (a round is any set of pairwise disjoint edges). [4] studies the problem of routing and call scheduling in 802.11 multihop wireless networks and provide an optimal framework for 
determining optimal routing and scheduling needed by the traffic in the network using a binary interference model and fixed transmission power. In [5], a joint scheduling, routing and power control strategy is proposed. The authors develop a computational tool using column generation to maximize the minimum throughput among all flows. They highlight the usefulness of the power control on the performance of multihop wireless networks.

The joint problem of power control and scheduling link transmissions in a wireless network in order to optimize performance objectives (throughput, delay, energy), received a lot of attention in the recent years [6]-[8]. In [6], the problem of finding a minimum-length schedule that satisfies a set of specified traffic demands is addressed, using a columngeneration-based solution method. It is shown that power control improves the spatial reuse, which leads to further improvement in schedule length compared to a fixed transmit power. Because of the complexity of scheduling with power control using a SINR model is NP-hardness [9], [10], several papers proposed heuristic algorithms to minimize the length schedule and the energy consumption with and without power control [9], [11].

To the best of our knowledge, only very few papers investigate on both the study of capacity and energy consumption. [12] studied energy, latency and capacity trade-offs existing in a multi-hop ad-hoc wireless network. The authors assume a linear topology with a simple energy model. They propose an analytical study that does not take into account a realistic interference model. The tradeoff between energy consumption and capacity is investigated using a binary interference model and a fixed transmission power in [2]. The relation between energy minimization and through-put maximization of a 802.11 WLAN is analyzed in [13].

\section{PROBLEM DEFINITION AND ASSUMPTIONS}

\section{A. Problem definition}

We focus on a fixed WMN based on Orthogonal Frequency Division Multiple Access (OFDMA) resource management. As in LTE-Advanced relay or WIMAX Mesh, a periodic frame is divided into time-slots. The bandwidth corresponding to a slot is subdivided into several blocks of 12 subcarriers, denoted as Physical Resource Block (PRB). The smallest resource unit that can be allocated to a BS is called Scheduling Block (SB) and covers 2 slots and 1 PRB. Note that this work can be applied to any synchronous slotted technology in which the resource is divided into time-frequency elements.

We assume that each base station is equipped with omnidirectional antennas. The transmission power can be adjusted from the minimum transmission level to the maximum one. All base stations periodically send a given quantity of traffic which represents the aggregate demand of their clients: these traffics require several scheduling blocks to be transmitted. The traffic is routed to gateways through multi-hop paths that will be computed by our linear program.

For a given available bandwidth for the backhauling network, our objective is to find an optimal schedule within a minimum time frame to maximize the capacity. Our framework allocates for each base station the optimal number of SB required to send its own traffic and to route the traffic of the other nodes.

\section{B. Network model and notations}

We assume a slotted, synchronized and static WMN, modeled as a directed graph $\mathrm{G}(\mathrm{V}, \mathrm{E})$. The set $\mathrm{V}_{\mathrm{BS}}$ represents the set of base stations, and $\mathrm{V}_{\mathrm{g}}$ the set of gateways $\left(\mathrm{V}=\mathrm{V}_{\mathrm{BS}} \cup \mathrm{V}_{\mathrm{g}}\right)$. Each $\mathrm{BS}$ of $\mathrm{V}_{\mathrm{BS}}$ routes to the gateway an aggregated demand $d_{v}$. We consider a stationary network, hence periodic, of period $\mathrm{T}$. The throughput of the WMN, defined as the ratio between the total traffic received at the gateways and the period length to collect it, is $\frac{\sum_{v} d_{v}}{T}$. Therefore, to optimize the throughput is to minimize the number of time slots used to activate the links transmitting the traffic. An insight of a throughput-optimal scheduling policy would be to pack as many links as possible in each time slot, that is maximizing the spatial reuse of system resources. This objective has to be mitigated with interference and energy consumption constraints.

The set of edges $\mathrm{E} \subseteq \mathrm{V} \times \mathrm{V}$ corresponds to the communication links. A link $(u, v) \in E$ exists if and only if the sender node $u$ can communicate directly with the receiver node $v$. We denote $(\mathrm{u}, \mathrm{v}, \mathrm{k})$ a transmission between nodes $\mathrm{u}$ and $\mathrm{v}$ on PRB $k$. This transmission is successful only if the SINR at the receiver exceeds the minimum threshold $\beta$. Let $P_{u}$ denotes the transmission power of the sender $u$. We assume that the received power in $v$ depends on the attenuation function, denoted $L(u, v)$, which depends on both the distance $d(u, v)$ and the environment. In this paper we will use the classical path-loss attenuation $L(u, v)=d(u, v)^{-\alpha}$ where $\alpha$ is the path loss exponent, but any other propagation model can be used. The SINR condition at receiver $v$ in the presence of other transmissions is expressed by the following equation:

$$
\operatorname{SINR}_{(u, v)}=\frac{P_{u} * L(u, v)}{\mu_{v}+\sum_{\left(u^{\prime}, v^{\prime}\right) \neq(u, v)} P_{u}^{\prime} * L\left(u^{\prime}, v\right)} \geq \beta_{v},
$$

where $\mu \in \mathbb{R}^{+}$represents the thermal noise at the receiver. A set of transmissions is feasible if the Eq. (1) is respected at all receiver. We define a configuration $\mathrm{F}$ as a set of transmissions that can be activated simultaneously in a time slot. The set of all possible configurations is denoted $\mathcal{F}$. Increasing the cardinality of a configuration $(F \in \mathcal{F})$ strengthens the spatial reuse of the links, which contributes to increase the throughput.

Communications are identified by the following physical parameters:

- $c_{(u, v)}^{k}$ is the capacity of the link $(u, v)$ on the PRB $k$.

- $J_{(u, v)}^{k}$ is the total energy consumed for communicating on the link $(\mathrm{u}, \mathrm{v}) . u$ spends a transmitting cost $j_{t}^{k}(u)$ while $v$ spends a receiving cost $j_{r}^{k}(v), J_{(u, v)}^{k}=j_{t}^{k}(u)+j_{r}^{k}(v)$.

\section{EEJCO-PC: ENERGY EFFICIENT JOINT CAPACITY OPTIMIZATION AND POWER CONTROL}

Maximizing the number of simultaneous transmissions allows to minimize the time frame (total slot number), i.e. to 
maximize the capacity. Unfortunately, it leads to an higher total transmission cost because maximizing the concurrent transmissions increases the interferences. On the other hand, power control mechanisms aim at minimizing the transmission powers which can save a significant energy.

A link $e=(u, v)$ is in the configuration $\mathrm{F}$ if and only if there exist at least one PRB $\mathrm{k}$ such as $(u, v, k) \in \mathrm{F}$. The capacity of the link $e$ in the configuration $\mathrm{F}$ is $c_{e}(\mathrm{~F})=$ $\sum_{k,(u, v, k) \in \mathrm{F}} c_{e}^{k}$.

A node $v$ which is involved in no active transmission is said idle and denoted $v \notin \mathrm{F}$, for sake of simplicity. The energy cost of an idle node $v$ is $J_{\text {idle }}(v)$.

Each feasible configuration $\mathrm{F}$ has an energy cost $J(\mathrm{~F})$ taking into account the active transmissions and the idle nodes: $J(\mathrm{~F})=\sum_{(u, v, k) \in \mathrm{F}}\left(j_{t}^{k}(u)+j_{r}^{k}(v)\right)+\sum_{v \notin \mathrm{F}} J_{\text {idle }}(v)$.

At each time, one and only one configuration is active and $w(\mathrm{~F})$ denotes the duration of activation of the configuration $\mathrm{F}$. The total length of the period is hence $\mathrm{T}=\sum_{F \in \mathcal{F}} w(\mathrm{~F})$ and the total communication cost is $\sum_{\mathrm{F} \in \mathcal{F}} w(\mathrm{~F}) J(\mathrm{~F})$.

\section{A. Routing and Scheduling}

The activation of a configuration $\mathrm{F}$ during a time unit provides to each link $e$ a capacity $c_{e}(\mathrm{~F})$. The total link capacity through the period is $\sum_{\mathrm{F} \in \mathbf{F}, \mathrm{F} \ni e} c_{e}(\mathrm{~F}) w(\mathrm{~F})$. This capacity is used to route the traffic from the mesh routers to the gateways.

For each node $u, \mathcal{P}_{u}$ denotes the set of all possible paths between $u$ and a gateway, and all the possible paths are $\mathcal{P}=$ $\cup_{u} \mathcal{P}_{u}$. The traffic flow on the path $\mathrm{P}$ is $\mathrm{f}(\mathrm{P})$. The traffic sent by $u$ is hence $\sum_{\mathcal{P}_{u}} f(\mathrm{P})$. The flow over a link $e$ is the sum of the traffic on the path going through $e, \sum_{\mathrm{P} \ni e} f(\mathrm{P})$. This flow has to be below the capacity of $e$.

The joint routing and scheduling problem is expressed in the two following linear programs (LP): one is capacity oriented, the other one is energy oriented. The first one maximizes the capacity with an energy budget constraint. Let us call the following LP the Master Problem to Maximize Capacity (MPMC):

$$
\begin{gathered}
\min \sum_{\mathrm{F}} w(\mathrm{~F}) \\
\text { subject to } \forall r \in V_{r} \quad \sum_{\mathrm{P} \in \mathcal{P}_{r}} f(\mathrm{P})=d(r) \\
\forall e \in E \quad \sum_{\mathrm{P} \in \mathcal{P}, \mathrm{P} \ni e} f(\mathrm{P}) \leq \sum_{\mathrm{F} \in \mathrm{F}, \mathrm{F} \ni e} c_{e}(\mathrm{~F}) w(\mathrm{~F}) \\
\sum_{\mathrm{F}} w(\mathrm{~F}) J(\mathrm{~F}) \leq J
\end{gathered}
$$

Equations (2)-(3) express the routing part as a flow from BSs to the gateway. Eq. (4) constrains the total energy expenditure of the network to a budget $J$ while the objective is to minimize the time frame, i.e. maximizing the capacity.

The energy oriented version minimizes the total energy expenditure subject to capacity guarantee. The flow equations are the same as Eq. (2)-(3) while Eq. (5) upper bounds the period length, i.e. lower bounding the capacity. Let us call the following LP the Master Problem to Minimize Energy consumption (MPME):

$$
\min \sum_{\mathrm{F}} w(\mathrm{~F}) J(\mathrm{~F})
$$

subject to Equations (2)-(3) and

$$
\sum_{\mathrm{F}} w(\mathrm{~F}) \leq T
$$

Because the number of paths and configurations is exponential with the size of the network, these formulations are not scalable as it is. Column generation [4], [5] is a prominent and efficient technique to cope with this situation. Based on sophisticated linear programing duality results, it allows to save the enumeration of the variable sets. The column generation description implemented is described below.

\section{Column Generation}

The idea of column generation is to solve a Master Problem with restricted sets of paths $\mathcal{P}_{0}$ and configuration $\mathrm{F}_{0} ; \mathcal{P}_{0}$ and $\mathcal{F}_{0}$ have to be carefully chosen to ensure the existence of an initial feasible solution. Generally, $\mathcal{P}_{0}$ should contain a shortest path from each base station to a gateway, and $\mathcal{F}_{0}=\{\{\mathrm{e}\}, \mathrm{e} \in \mathrm{E}\}$. Solving the Master Program generates a set of dual values, described in the following section. Given these values, auxiliary programs described in Section V-B, seek a column of the master program (i.e a path or a configuration) violating the corresponding equation of the dual. If such a column exists it may improve the solution, the master program is hence solved using it, and the process loops.

\section{A. Dual formulation}

Due to the lack of space, we present only the dual formulation of MPMC, the one of MPME being very similar. In this LP, there is a constraint corresponding to each path or configuration variable of the master. We denote $\theta(r), \forall r \in \mathrm{V}_{\mathrm{BS}}$ the dual variable associated with constraint Eq. (2), $\lambda(e), \forall e \in \mathrm{E}$ associated with constraint Eq. (3) and $\sigma$ associated with constraint Eq. (4). $\mathrm{O}(\mathrm{P})$ denotes the source node of path P. $J_{u}$ is the energy consumption of node $u$ which depends on its activity: transmission, reception, or idle.

\section{Dual formulation of MPMC:}

$$
\max \sum(\theta(r) d(r))-\sigma J
$$

$$
\begin{gathered}
\text { subject to: } \quad \forall \mathrm{P} \in \mathcal{P} \quad \theta(\mathrm{O}(\mathrm{P})) \leq \sum_{e \in \mathrm{P}} \lambda(e) \\
\forall \mathrm{F} \in \mathcal{F} \quad \sum_{e} \sum_{k} c_{e}^{k} \lambda(e)-\sigma \sum_{u} J_{u} \leq 1
\end{gathered}
$$




\section{B. Auxiliary problems}

The auxiliary problems determine if there are paths or configurations that violate the constraints of the dual program. The column generation algorithm involves two such problems, one for each kind of dual constraints. The first one, associated to constraint Eq. (6), finds, for each source node, a weighted path with a weight lower than the dual variable associated to the source node. If the minimum weighted path fits the constraint then all other paths do. This problem is hence solved by any shortest path algorithm or linear program.

The second auxiliary problem is associated to constraint Eq. (7). We define a configuration as $\sum_{e} \sum_{k} c_{e}^{k} \lambda(e)-\sigma \sum_{u} J_{u}>$ 1. Again, if the maximum weight communication set respects Eq. (7) then all other configurations do.

Given a topology, the problem is hence to find a configuration $\mathrm{F} \in \mathcal{F}$ where $\sum_{e} \sum_{k} c_{e}^{k} \lambda(e)-\sigma \sum_{u} J_{u}$ is maximum on $\mathrm{F}$. To compute such a configuration, with SINR interferences and energy consumption models, we develop the following Mixed Integer Linear Program. The SINR requirements are modeled by constraint Eq. (13). The energy consumption model takes into account the cost of the nodes transmitting or receiving Eq. (9), and the consumption of idle nodes (10).

$$
\begin{gathered}
\max \sum_{e \in E} \sum_{k}\left(c_{e}^{k} \lambda_{e}\right)-\sigma \sum_{u} J_{u} \\
\forall u, v, k \quad J_{u} \geq \sum_{k} T_{s} * P_{t}^{k}(u)+\sum_{k} \sum_{v} j_{r}^{k}(v) \Psi_{(v, u), k} \\
\forall u \quad J_{u} \geq J_{\text {idle }}(u) \\
\forall e=(u, v), k \quad c_{e}^{k}=C_{1} \Psi_{(u, v), k} \\
\forall u, k \quad \sum_{v} \psi_{(u, v), k}+\sum_{w} \psi_{(w, u), k} \leq 1 \\
\forall u, v, k \quad P_{t}^{k}(u) * L(u, v) \geq \beta *\left(\sum_{\left(u^{\prime}, v^{\prime}\right) \neq(u, v)} P_{t}^{k}\left(u^{\prime}\right) * L(u, v)\right. \\
+\mu)-\left(1-\psi_{(u, v), k}\right) n P_{\max }
\end{gathered}
$$

$$
\forall u, k \quad P_{t}^{k}(u) \leq P_{\max }
$$

$\psi_{(u, v), k}$ is a binary variable associated with the link (u,v) at the PRB k. If $\psi_{(u, v), k}=1$ then the link (u,v) is active and the PRB $\mathrm{k}$ in the new configuration.

\section{RESULT ANALYSIS AND DISCUSSION}

Both the capacity-oriented (MPMC) and energy-oriented (MPME) formulations, as well as the column generation algorithm have been implemanted using AMPL/CPLEX. We have considered network topologies composed of $25 \mathrm{BSs}$ with a gateway located at the center of the network. The distance between adjacent BSs is set to 83 meters (Small Cell). The radio parameters of LTE system are used: the SB covers $1 \mathrm{~ms}$ (2 slots) and 1 PRB (180 khz). For the attenuation function, the path-loss exponent $(\alpha)$ equals to 2.6. The transmission power varies between 0 and $0.08 \mathrm{~W}$. We assume that all BSs have the same traffic demands.

\section{A. Impact of physical layer parameters}

We investigate the sensitivity of the network capacity and the energy consumption to the SINR threshold variation (from 1 to 70). For each SINR value, the minimum time frame and the total energy consumption are reported on Fig. 2. One can remark that the time frame is a step function while the energy consumption grows at each step. Considering the impact of the SINR on a given configuration gives an insight on this behavior. Let us consider an admissible configuration with SINR 1. Increasing the SINR threshold, all links can be kept active by increasing the transmission power to mitigate the sensitivity to interferences. This energy cost explodes as the interferences get too strong. Once this step is reached, some links have to be deactivated which results in an increase of the time frame and a decrease of the energy consumption.

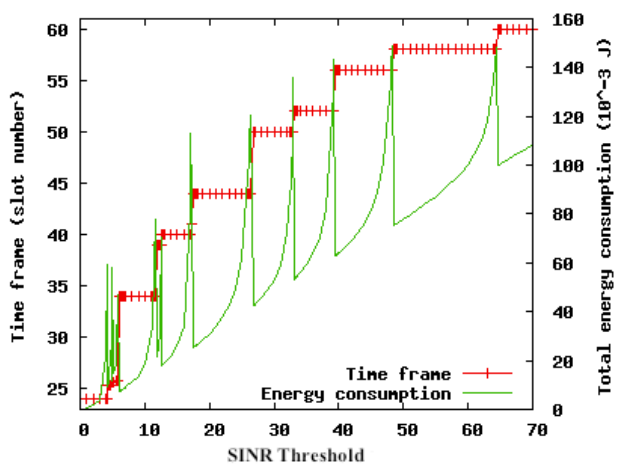

Fig. 2. Energy consumption and time frame vs SINR threshold.

In Fig. 3, we study the same scenario under the following assumptions: the SINR threshold is set to 10 , the idle node consumption equals to $40 \%$ of the reception cost, the path loss exponent varies between 2 (ideal empty 2D space) and 4.5 (indoor environment with many obstacle or very dynamic). It shows an exponential growth of the overall energy consumption. BS position have significant impact on the energy consumption: a BS located in a perturbed environment consume much more than one situated in a ideal environment.

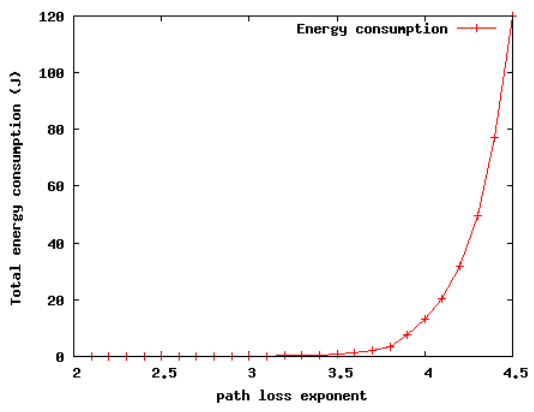

Fig. 3. Energy consumption vs path loss exponent: : Idle $=0.4 * \mathrm{RX}$.

\section{B. Capacity and energy tradeoff}

The Pareto front of the capacity/energy tradeoff, when the consumption of idle nodes is null, is depicted in Fig. 4. Note 
the requirement of a minimal energy budget for the network to route all the traffic demand. Optimizing the capacity needs to maximize the spatial reuse in the configurations, with a transmission power cost in order to mitigate the resulting interferences. This is confirmed by the evolution of the mean cost of a transmission with the density of the configurations, reported in Fig. 6 (see link_cost without idle cost).

We also investigate an idle energy consumption varying from $20 \%$ to $100 \%$ of the reception cost. It adds a penalty on the energy consumption for each non transmitting node, which is an energetic incentive for spatial reuse. Consequently, the strategy for minimizing the energy consumption is twisted to the one for increasing the capacity. The magnitude of the energy-capacity tradeoff corresponding to the idle energy cost is reported as Fig 5. As expected, the tradeoff disappears as the consumption of idle nodes grows. Again, the behavior of the mean cost of a transmission with the configurations density, reported in Fig. 6, confirms this fact. Indeed, the total consumption of the idle nodes is shared among the cost of the active transmissions, and this total reduces as the cardinality of the configuration increases. When the consumption of idle nodes is significative ( $20 \%$ is enough), the mean cost of a transmission decreases with the density of the configurations.

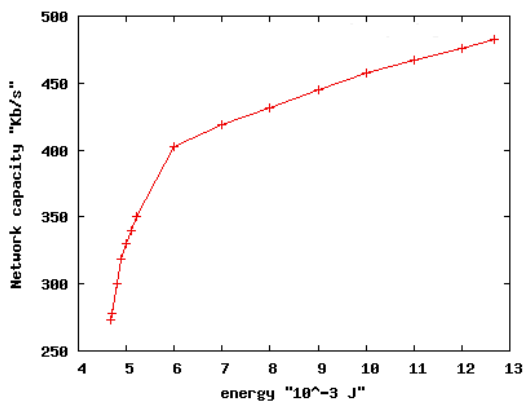

Fig. 4. Capacity and energy tradeoff, assuming Idle cost $=0$.

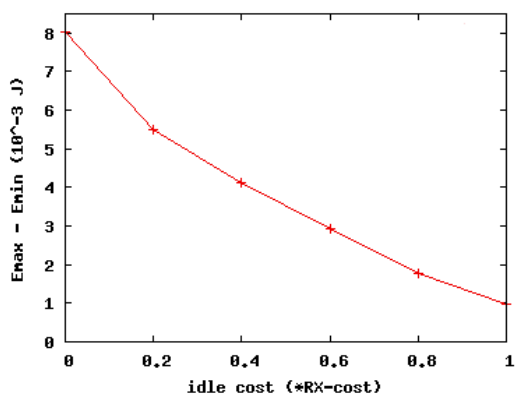

Fig. 5. Capacity and energy tradeoff.

\section{CONCLUSION}

We have presented a column generation algorithm which computes a linear relaxation of the Routing and call Scheduling Problem with a realistic SINR model and power control. This tool can be used in any WMN where the resource is

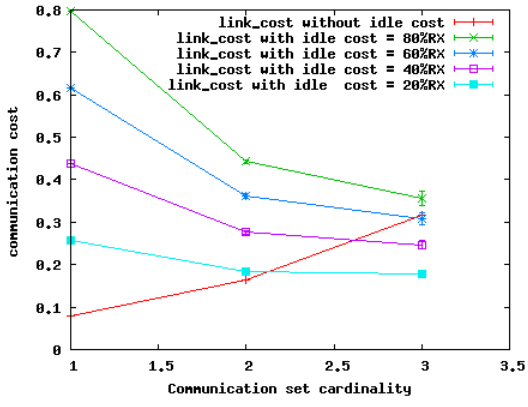

Fig. 6. Communication cost vs transmission set size.

divided into time-frequency elements. We have investigated the energy-capacity tradeoff, and the impact of some physical layer parameters on these metrics. We showed that the capacity and energy efficiency can be jointly maximized when the power consumption of idle nodes is important. A deeper challenge is to build an actual assignment of the resources, based on the weighting computed, and taking into account delay constraints and dynamic traffic demands.

\section{ACKNOWLEDGMENT}

This work has been partially funded by the ANR VERSO project ECOSCells.

\section{REFERENCES}

[1] P. Gupta and P. R. Kumar. The capacity of wireless networks. IEEE Transactions on Information Theory, vol. 46:388-404, March 2000.

[2] A. Ouni, H. Rivano, and F. Valois. Column generation for studying capacity and energy trade-off in LTE-like network. Technical report, INRIA, RR-7437, October 2010.

[3] J-C. Bermond, J. Galtier, R. Klasing, N. Morales, and S. Pérennes. Hardness and approximation of gathering in static radio networks. Parallel Processing Letters, 16(2):165-183, 2006.

[4] C. Molle, F. Peix, and H. Rivano. An optimization framework for the joint routing and scheduling in wireless mesh networks. PIMRC '08, Cannes, France, September 2008.

[5] J. Luo, C. Rosenberg, and A. Girard. Engineering wireless mesh networks: Joint scheduling, routing, power control and rate adaptation. IEEE/ACM Transactions on Networking, April 2009.

[6] S. Kompella, J.E. Wieselthier, A. Ephremides, H.D. Sherali, and G.D. Nguyen. On optimal sinr-based scheduling in multihop wireless networks. IEEE/ACM Transactions on Networking, pp. 1713 - 1724, December 2010.

[7] T. ElBatt and A. Ephremides. Joint scheduling and power control for wireless ad hoc networks. IEEE transactions on wireless communications, vol. 3, no1, pp. 74-85, January 2002.

[8] R. Bhatia and M. Kodialam. On power efficient communication over multi-hop wireless networks: joint routing, scheduling and power control. INFOCOM 2004, 1457 - 1466 vol.2, March 2004.

[9] K. Bastian, V. Markus, and W. Dorothea. Energy efficient scheduling with power control for wireless networks. In WiOpt'10, Avignon, France, June 2010.

[10] O. Goussevskaia, Y. A. Oswald, and R. Wattenhofer. Complexity in geometric sinr. ACM MobiHoc '07, pp. 100-109, 2007.

[11] Gang Lu and Bhaskar Krishnamachari. Energy efficient joint scheduling and power control for wireless sensor networks. In IEEE SECON '05, pages 362-373, 2005.

[12] J-M Gorce, R. Zhang, K Jaffrès-Runser, and C. Goursaud. Energy, latency and capacity trade-offs in wireless multi-hop networks. In PIMRC '10, Istanbul, Turkey, September 2010.

[13] P. Serrano, M. Hollick, and A. Banchs. On the trade-off between throughput maximization and energy consumption minimization in ieee 802.11 wlans. EURASIP Journal on Wireless Communications and Networking, April 2010. 